\title{
SYMMETRIZING KERNELS AND THE INTEGRAL EQUATIONS OF FIRST KIND OF CLASSICAL POTENTIAL THEORY
}

\section{JAMES LUCIEN HOWLAND}

1. A symmetric kernel $G$ is said to symmetrize the kernel $K$ by composition on the left in case the product $G K$ is symmetric-i.e. in case $G K=K^{T} G$. It follows at once that, if $G$ is a left symmetrizer of $K$, so are $G K, G K^{2}, G K^{3}$, etc., and that the linear manifold spanned by these kernels consists entirely of left symmetrizers of $K$. It also follows that $G^{-1}$, when it exists, satisfies $K G^{-1}=G^{-1} K^{T}$, so that the inverse of $G$ is to be sought among the right symmetrizers of $K$.

The integral equations of first kind of classical potential theory, namely

$$
\begin{aligned}
& f(p)=\int_{S} G(p q) \mu(q) d S_{q}, \\
& g(p)=\int_{S} D(p q) \nu(q) d S_{q}
\end{aligned}
$$

arise when the solution of the Dirichlet problem with respect to a surface $S$ is sought in the form of the potential $V[\mu]$ of a surface distribution on $S$ of density $\mu$, and the solution of the Neumann problem is sought in the form of the potential $W[\nu]$ of a double layer on $S$ of moment $\nu$. In this notation, $G(p, q)=1 /\left(2 \pi r_{p q}\right)$ is the potential at $p(q)$ of a unit mass at $q(p)$, and is symmetric, while $D(p, q)$ $=\left(\partial^{2} / \partial n_{p} \partial n_{q}\right) G(p q)$ represents the normal component of force at $p(q)$ due to a unit normal dipole at $q(p)$ and is likewise symmetric. The given boundary values relevant to the (interior or exterior) Dirichlet and Neumann problems are $f(p)$ and $g(p)$, respectively.

In this paper, the concepts of the first paragraph above are applied to the solution of the equations (1) and (2) in the case of a closed, bounded surface $S$ of class $B[11$, p. 186]. For, it is known [19, $\S 4$, p. 344] that $G$ is a left symmetrizer of the kernel

$$
K(p, q)=\left(\partial / \partial n_{p}\right) G(p, q)=\cos \left(r, n_{p}\right) /\left(2 \pi r_{p q}^{2}\right)
$$

of the Fredholm-Poincaré integral equations. It will be shown that

$$
D(p, q)=\left[\cos \left(n_{p}, n_{q}\right)+3 \cos \left(n_{p}, r\right) \cos \left(n_{q}, r\right)\right] /\left(2 \pi r_{p q}^{3}\right)
$$

Presented to the Society, November 25, 1966 under the title Symmetrizing kernels and the integral equations of first kind of potential theory; received by the editors November 8, 1966. 
is a right symmetrizer of $K$ and that $D G=K^{2}-I$ so that, in effect $G^{-1}=-\left[D+K^{2} D+K^{4} D+\cdots\right], \quad D^{-1}=-\left[G+G K^{2}+G K^{4}+\cdots\right.$ । and the integral equations (1) and (2) are equivalent, respectively, to

$$
\begin{aligned}
K^{2} \mu-\mu & =D f, \\
\left(K^{T}\right)^{2} \nu-\nu & =G g .
\end{aligned}
$$

Analogous results may be obtained in the plane.

The problem represented by the integral equation (1) has been discussed by Liapounoff [10] who showed that, when $f$ is such that $W[f]$ admits a regular normal derivative on $S$, it may be written $f=G \mu$. The density $\mu$ is obtained as the difference of the solutions of two in tegral equations, formulated with respect to the regions interior and exterior to $S$, respectively. For general $f$ he showed that the third and succeeding terms of the Neumann series solution of the Dirichlet problem with boundary values $f$ could be written as the potential of a single layer but, in general, the first two terms could not. Similar results were obtained by E. R. Neumann [14] who, in addition, obtained the solution of the Neumann problem in the form of a double layer potential [14, pp. 43-66]-i.e. solved the integral equation (2). Bertrand [1] converted the equation (1), in the two-dimensional case, to an equation of second kind by differentiation while Plume [21] has given a similar treatment of the Neumann problem. Picard [17], [18], in a well-known paper, has given necessary and sufficient conditions, applicable in the two-dimensional case, that (1) admit a square-integrable solution, and has worked out the case of a circle. These methods have been extended to the three-dimensional case by Fenyo [3], who illustrates his results in the case of a sphere. Blumer [2] converts (1), in the three-dimensional case, to each of three integral equations of second kind by a complicated process based upon integro-differential operators analogous to those of M. Riesz. Thus it appears that the symmetrizing property of $D$ and the equivalence of (1) and (2) with equations of second kind with kernels $K^{2}$ and $\left(K^{T}\right)^{2}$ are new results. The following development, however, owes much to the work of Liapounoff [8], [9], [10].

2. A function $V(P)$ harmonic in the region $R$ interior, or $R^{\prime}$ exterior, to $S$ is said to possess a regular normal derivative on $S[9, \S 2$, p. $246, \$ 16$, p. 285] in case $\lim _{P \rightarrow p \in S}(\partial V / \partial n)(P)$ is taken uniformly on $S$ as $P \rightarrow p$ along the normal to $S$ at $p$. (The normal $n$ is defined throughout as the interior normal to $S$, and determines the positive (interior) and negative (exterior) sides of $S$. See, e.g. equations (6) 
and (8).) These limiting values then define a continuous function on $S$. Tauber [22], [23] (see also Liapounoff [8, p. 131]) has shown that the difference of the derivatives of a double layer potential $W[\nu]$, with continuous moment $\nu$ on $S$, in the normal direction at points on the normal on opposite sides and equidistant from $S$, vanishes as these points approach $S$, and hence that, if $W[\nu]$ admits a regular normal derivative on one side of $S$, it does so on the other side, and the limiting values of the normal derivatives are equal. Gunther [6, p. 70] has quoted an example of a surface $S$ and continuous function $\nu$ such that $W[\nu]$ does not admit a regular normal derivative. Analytic conditions sufficient for the existence of a regular normal derivative $D \nu$ of $W[\nu]$ have been formulated by C. Neumann $[12$, p. 413], [13, p. 436], Liapounoff $[8$, p. 132], [9, \$20, p. 293 et seq.], and Kellogg [7, p. 42 et seq.] while complicated necessary and sufficient conditions have been established by Petrini [15, p. 320], $[16$, p. 212]. Liapounoff $[9, \S 19$, p. 293] has characterized the domain of $D$ by showing that, for continuous $\nu, W[\nu]$ admits a regular normal derivative on $S$ when, and only when, the solution of the Dirichlet problem with respect to $R$, determined by the boundary values $\nu$, also admits a regular normal derivative on $S$.

Liapounoff $[9, \S \S 15,16]$ (see also Plemelj $[20, \$ 4$, p. 9]) has established the following extension of Green's third identity:

Lemma. Suppose that $V(P)$ is harmonic in $R$ and admits a regular normal derivative on $S$. Then, when $P \in R$,

$$
\chi(P)=\frac{1}{2} W[V]-\frac{1}{2} V[\partial V / \partial n]=V(P)
$$

and, when $P \in R^{\prime}, \chi(P)=0$.

It follows at once from (5), together with the formulae

$$
\left.W\right|_{+}=\nu+K^{T} \nu,\left.\quad W\right|_{-}=-\nu+K^{T} \nu,
$$

describing the discontinuity in $W[\nu]$ across $S$, that, on $S$,

$$
V=K^{T} V-G(\partial V / \partial n) \text {. }
$$

Moreover, if it is assumed that $W[V]$ admits a regular normal derivative on $S$, it follows from (5) and the formulae

$$
\left.(\partial V / \partial n)\right|_{+}=-\mu+K \mu,\left.\quad(\partial V / \partial n)\right|_{-}=\mu+K \mu
$$

describing the discontinuity in the normal derivative of $V[\mu]$ across $S$ that, on $S$,

$$
\partial V / \partial n=D V-K(\partial V / \partial n) .
$$


The formulae (7) and (9) may be interpreted as integral equations connecting the limiting values $V$ and $\partial V / \partial n$ on $S$. It is important to observe that (7) is the Neumann-Poincare integral equation in the case $\lambda=+1$, corresponding to the exterior Dirichlet problem, while (9) is the Robin-Poincare integral equation in the case $\lambda=-1$, corresponding to the exterior Neumann problem. The questions of existence and uniqueness of solutions of these equations have been discussed in detail by Plemelj $[19, \S 16$, p. 383 et seq. $]$. In particular, it is known that $\lambda=-1$ is not an eigenvalue of $K$ and that (9) possesses an unique, continuous solution for any nonhomogeneous term $D V$.

3. The characteristic properties of $G$ and $D$ now follow at once. For, whenever $\mu$ is continuous on $S$, substitution from the first of equations (8) into (7) is permissible and leads directly to the formula (see also Plemelj, loc. cit.)

$$
G K \mu=K^{T} G \mu .
$$

Similarly, since $V[\mu]$ admits a regular normal derivative on $S$, so does $W[V]$, and substitution from the first of equations (8) into (9) is also permissible, to obtain

$$
D G \mu=K^{2} \mu-\mu .
$$

When $\nu$ is continuous on $S, W[\nu]$ may be represented in $R$ as the sum $W[\nu]=V_{1}(P)+V_{2}(P)$ of two harmonic functions characterized by the boundary values $\nu$ and $K^{T} \nu$ on $S$, respectively. When $W[\nu]$ admits a regular normal derivative on $S$, so does $V_{1}(P)$, whence $V_{2}(P)$ does also, and so $W\left[K^{T} \nu\right]$ does also. Thus $D$ and $D K^{T} \nu$ both exist, and $D\left(\nu+K^{T} \nu\right)=D \nu+D K^{T} \nu$. Substituting, then, from the first of equations (6) into (9), and applying this relation, the formula

$$
D K^{T} \nu=K D \nu
$$

is obtained. Similar substitution into (7) leads to

$$
G D \nu=\left(K^{T}\right)^{2} \nu-\nu .
$$

4. It is well known (Plemelj, loc. cit. $\$ 2$ ) that $\lambda=+1$ is an eigenvalue of the kernel $K$ of the Fredholm-Poincare integral equations, and that, correspondingly, the homogeneous equations $K^{T} \nu_{1}-\nu_{1}=0$, $K \mu_{1}-\mu_{1}=0$ each admit a single eigenfunction. The eigenfunction $\nu_{1}$ is constant, while $\mu_{1}$ represents the equilibrium distribution of charge on $S$. It follows from (10) that, with appropriate normalization, $\nu_{1}=G \mu_{1}$. On the other hand, since $\mu_{1}$ is continuous, $V\left[\mu_{1}\right]$ has a regular normal derivative on $S$ whence, $W[V]=W\left[\nu_{1}\right]$ has also. However, $D \nu_{1}=0 \neq \mu_{1}$. 
The identities $K^{2} \mu-\mu=K \mu-\mu+K(K \mu-\mu)$ and $\left(K^{T}\right)^{2} \nu-\nu=K^{T} \nu$ $-\nu+K^{T}\left(K^{T} \nu-\nu\right)$, together with the fact that $\lambda=-1$ is not an eigenvalue of $K$ or $K^{T}$, show that $\mu_{1}$ and $\nu_{1}$ are also the only eigenfunctions of $K^{2}$ and $\left(K^{T}\right)^{2}$ respectively. Thus, it follows from (13) that $D \nu=0$ implies $\nu=$ constant.

These remarks, together with Fredholm's third theorem, show that the integral equations (3) and (4) admit solutions when, and only when, $\int D f d S=0$ and $\int g G \mu_{1} d S=0$, respectively; and that these solutions are not unique but contain an added arbitrary multiple of the corresponding eigenfunction.

5. Theorem 1. A necessary and sufficient condition that the integral equation (1) shall admit an unique continuous solution $\mu$ for any given continuous function $f$ is that $f$ lie in the domain of $D$. When this is the case, $\mu$ satisfies the equation (3).

Proof. 1. When $\mu$ is a continuous solution of (1), $V[\mu]$ admits a regular normal derivative on $S$ and, thus, so does $W[f]$. From (8)and (9) it follows that $\mu$ satisfies (3).

2. When $D f$ exists, equation (3) may be formulated and, since $\int_{S} D f d S=0$ this equation admits a continuous solution $\mu=\mu_{0}+C \mu_{1}$. For each such solution it follows from (1) that $D G \mu=D f$, whence $G \mu-f=G \mu_{0}+C G \mu_{1}-f$ is constant on $S$. But $G \mu_{1}=\nu_{1}$ is itself constant, thus $C$ may be uniquely chosen such that (1) is satisfied. Q.E.D.

Theorem 2. A necessary and sufficient condition that the integral equation (2) shall admit a continuous solution $\nu$, unique to within an additive constant, for any given continuous function $g$, is

$$
\int_{S} g d S=0 .
$$

When this is the case, $\nu$ satisfies the equation (4) or, alternatively, $\nu=G \mu$ where $\mu$ satisfies the equation (3) with Df replaced with $g$.

Proof. 1. When $\nu_{0}$ satisfies (2), so that $D \nu_{0}=g=\partial / \partial n W\left[\nu_{0}\right]$ then $g$ satisfies (14). Moreover, from (13), $G D \nu_{0}=G g=\left(K^{T}\right)^{2} \nu_{0}-\nu_{0}$, whence $\nu_{0}$ satisfies (4). Since $\nu_{0}$ lies in the domain of $D, \nu_{0}=G \mu$ for some continuous $\mu$, and $\mu$ satisfies (3) with $D f$ replaced with $g$, by Theorem 1 . These same conclusions are clearly valid for $\nu=\nu_{0}+C \nu_{1}$, which also satisfies (2).

2. Given a continuous function $g$ satisfying (14), it follows that $\int \mu_{1} G g d S=\int \mu_{1} g d S=\nu_{1} \int g d S=0$, whence (4) possesses a continuous solution $\nu=\nu_{0}+C \nu_{1}$. Similarly (3) with $D f$ replaced with $g$ possesses a continuous solution $\mu=\mu_{0}+C \mu_{1}$, whence $G \mu=G \mu_{0}+C G \mu_{1}=G \mu_{0}+C \nu_{1}$ 
and it follows from (10) that $\nu=G \mu$ and that every solution of (4) has this form. Thus, $W[\nu]$ has a regular normal derivative on $S$, and from (13) it follows that $G D \nu=G f$, whence $D \nu=f$. Q.E.D.

Since $W\left[\nu_{1}\right]$ is constant in $R$ and zero in $R^{\prime}$ this theorem is in accordance with the known fact that the Neumann problem possesses an unique, regular solution in $R^{\prime}$, but that the solution is only determined to within an additive constant in $R$.

A second integral equation may, in certain circumstances, be formulated for the Dirichlet problem as follows:

Corollary. Suppose that $\int f \mu_{1} d S=0$. Then, the solution $\mu$ of (1) and (3) may be written $\mu=D \nu$ where $\nu$ is a continuous solution of (4) with $G g$ replaced with $f$.

Proof. 1. When $\mu$ satisfies (1), $0=\int \mu_{1} f d S=\int \mu_{1} G \mu d S=\int \nu_{1} \mu d S$ $=\nu_{1} \int \mu d S$ so that, by Theorem $2, D \nu=\mu$ possesses a continuous solution $\nu$. This function satisfies (4) with $G g$ replaced with $f$.

2. Following the arguments of Theorem 2, it is seen that (4), with $G g$ replaced with $f$, possesses continuous solutions $\nu$ for which $W[\nu]$ admits an unique normal derivative on $S$. From (13), $G D \nu=f$ whence $\mu=D \nu$ is the solution of (1).

Q.E.D.

\section{REFERENCES}

1. G. Bertrand, Le problème de Dirichlet et le potentiel de simple couche, Bull. Sci. Math. (2) 47 (1923), 282-288, 298-307.

2. $\mathrm{H}$. Blumer, Beziehungen zwischen speziellen linearen Integralgleichungen erster und zweiter Art und Lösung des Dirichletschen Problems durch das Potential einer einfachen Schicht, Comment Math. Helv. 28 (1954), 197-224.

3. S. Fenyo, Über das Dirichletsche Problem beziiglich der Kugel, Publ. Math. Debrecen 3 (1953), 71-80.

4. I. Fredholm, Sur une nouvelle méthode pour la résolution du problème de Dirichlet, Öfversigt Kungl. Vetenskapsakad. Stockholm (1900), 39-46.

5. - Sur une classe d'equations fonctionelles, Acta Math. 27 (1903), 365-390.

6. N. Gunther, La théorie du potentiel et ses applications aux problemes fondamentaux de la physique mathématique, Gauthier-Villars, Paris, 1934.

7. O. Kellogg, Potential functions on the boundary of their regions of definition, Trans. Amer. Math. Soc. 9 (1908), 39-50.

8. A. Liapounoff, Sur le potentiel de la double couche, Comm. Soc. Math. Kharkov 6 (1897), 129-138.

9. - Sur certaines questions qui se rattachent au probleme de Dirichlet, J. Math. Pures Appl. (5) 4 (1898), 3, 241-311.

10. - - Sur le principe fondamental de la méthode de Neumann dans le problème de Dirichlet, Comm. Soc. Math. Kharkov (2) 7 (1902), 229-252.

11. L. Lichtenstein, Neure Entwicklung der Potentieltheorie, Konforme Abbildung. Encyklop. math. Wiss. Vol. II C 3 (1918), 177-377.

12. C. Neumann, Neue Sätze ïber das Logarithmische Potential, Math. Ann. 16 (1882), 409-431. 
13. - Neue Sätze das Newton' sche Potential, Math. Ann. 16 (1882), 432-438.

14. E. Neumann, Beitrage zu einzelen Fragen der höheren Potentialtheorie, Teubner, Leipzig, 1912.

15. H. Petrini, Les derivées premières et secondes du potentiel, Acta Math. 31 (1908), $127-332$.

16. - Les derivées premières et secondes du potentiel logarithmique, J. Math. Pures Appl. (6) 5 (1909), 127-223.

17. E. Picard, Quelques remarques sur les équations intégrales de première éspèce et sur certains problèmes de physique mathématique, C. R. Acad. Sci. Paris 148 (1909), 1563-1568.

18. - Sur un théorème général relatif aux équations intégrales de première éspèce et sur quelques problèmes de physique mathématique, Rend. Circ. Mat. Palermo 29 (1910), 79-97.

19. J. Plemelj, Über Lineare Randwertaufgaben der Potentialtheorie, Monatsh. Mat. Physik 15 (1904), 337-411.

20. —, Potentialtheoretische Untersuchungen, Teubner, Leipzig, 1911.

21. Z. Plume, Potencialteorijas neimana problemas atrisināšana ar singularo integralvienadojumu metodi, Latvijas PSR Zinātṇu Akad. Vēstis (37) 8 (1950), 119126.

22. A. Tauber, Über das Potential einer Doppelbelegung, Monatsh. Math. Physik 8 (1897), 79-86.

23. —Über Einige Sätze der Potentialtheorie, Monatsh. Math. Physik 9 (1898), 74-88.

UNIVERSITY OF OTTAWA 